\title{
Ioana's Superrigidity Theorem and Orbit Equivalence Relations
}

\author{
Samuel Coskey \\ Department of Mathematics, Boise State University, 1910 University DR, Boise, ID 83725, USA \\ Correspondence should be addressed to Samuel Coskey; scoskey@nylogic.org
}

Received 9 October 2013; Accepted 10 November 2013

Academic Editors: M. Przybylska and A. Rapinchuk

Copyright (C) 2013 Samuel Coskey. This is an open access article distributed under the Creative Commons Attribution License, which permits unrestricted use, distribution, and reproduction in any medium, provided the original work is properly cited.

\begin{abstract}
We give a survey of Adrian Ioana's cocycle superrigidity theorem for profinite actions of Property (T) groups and its applications to ergodic theory and set theory in this expository paper. In addition to a statement and proof of Ioana's theorem, this paper features the following: (i) an introduction to rigidity, including a crash course in Borel cocycles and a summary of some of the best-known superrigidity theorems; (ii) some easy applications of superrigidity, both to ergodic theory (orbit equivalence) and set theory (Borel reducibility); and (iii) a streamlined proof of Simon Thomas's theorem that the classification of torsion-free abelian groups of finite rank is intractable.
\end{abstract}

\section{Introduction}

In the past fifteen years superrigidity theory has had a boom in the number and variety of new applications. Moreover, this has been coupled with a significant advancement in techniques and results. In this paper, we survey one such new result, namely, Ioana's theorem on profinite actions of Property (T) groups and some of its applications in ergodic theory and in set theory. In the concluding section, we highlight an application to the classification problem for torsion-free abelian groups of finite rank. The narrative is strictly expository, with most of the material being adapted from the work of Adrian Ioana, mine, and Simon Thomas.

Although Ioana's theorem is relatively recent, it will be of interest to readers who are new to rigidity because the proof is natural and there are many immediate applications. Therefore, we were keen to keep the nonexpert in mind. We do assume that the reader is familiar with the notion of ergodicity of a measure-preserving action and with unitary representations of countable groups. We will not go into great detail on Property $(\mathrm{T})$, since for our purposes it is enough to know that $\mathrm{SL}_{n}(\mathbb{Z})$ satisfies Property $(\mathrm{T})$ when $n>2$. Rather, we will introduce it just when it is needed, and hopefully its key appearance in the proof of Ioana's theorem will provide some insight into its meaning.

The concept of superrigidity was introduced by Mostow and Margulis in the context of studying the structure of lattices in Lie groups. Here, $\Gamma$ is said to be a lattice in the (real) Lie group $G$ if it is discrete and $G / \Gamma$ admits an invariant probability measure. Very roughly speaking, Margulis showed that if $\Gamma$ is a lattice in a simple (higher-rank) real Lie group $G$, then any homomorphism from $\Gamma$ into an algebraic group $H$ lifts to an algebraic map from $G$ to $H$. This implies Mostow's theorem, which states that any isomorphic lattices $\Gamma, \Lambda$ in a simple (higher-rank) Lie group $G$ must be conjugate inside $G$.

We will leave this first form of rigidity on the back burner and primarily consider instead a second form, initially considered by Zimmer, which is concerned with group actions. (The connection between the two forms of rigidity is that both can be cast in terms of measurable cocycles, which will be introduced in the next section. For the connection between cocycles and lifting homomorphisms, see [1, Example 4.2.12].) The basic notions are as follows. Two probability measure-preserving actions $\Gamma \curvearrowright X$ and $\Lambda \curvearrowright Y$ are said to be orbit equivalent if there exists a measure-preserving almost bijection $f: X \rightarrow Y$ such that $\Gamma x=\Gamma x^{\prime}$ if $\Lambda f(x)=\Lambda f\left(x^{\prime}\right)$. They are said to be isomorphic if additionally there exists an isomorphism $\phi: \Gamma \rightarrow \Lambda$ such that $f(\gamma x)=\phi(\gamma) f(x)$. Essentially, Zimmer showed that any (irreducible) ergodic action $\Gamma \curvearrowright X$ of a lattice in a (higher rank) simple Lie group is superrigid in the sense that it cannot be orbit equivalent to another action of an algebraic group $\Lambda \curvearrowright Y$ without being isomorphic to it. (For elementary reasons it is necessary to 
assume that $\Lambda$ acts freely on $Y$.) See [1, Theorem 5.2.1] for a weak statement of this result and [2, Section 1] for further discussion.

It is natural to ask whether there exists an analog of Zimmer's theorem in the context of general measure-preserving actions, that is, with the algebraic hypothesis on $\Lambda$ removed. Many rigidity results have been established along these lines (for instance, see [2-4]). One of the landmark results in this direction was obtained recently by Popa [5], who found a large class of measure-preserving actions $\Gamma \curvearrowright X$ which are superrigid in the general sense that $\Gamma \curvearrowright X$ cannot be orbit equivalent to another (free) action without being isomorphic to it. In particular his theorem states that if $\Gamma$ is a Property (T) group, then the free part of its left-shift action on $X=2^{\Gamma}$ (the so-called Bernoulli action) is an example of a superrigid action. Following on Popa's work, Ioana's theorem gives a second class of examples of superrigid actions, namely, the profinite actions of Property (T) groups.

This paper is organized as follows. The second section gives some background on Borel cocycles, a key tool in rigidity theory. A slightly weakened version of Ioana's theorem is stated in the third section. The proof itself is split between Section 4, which contains a general purpose lemma, and Section 5, which contains the heart of the argument. Although these are largely unchanged from Ioana's own account, I have inserted many additional remarks to smooth the experience for the newcomer.

In Section 6, we give a couple of the easier applications of the main theorem. First, we show how to obtain many orbit inequivalent profinite actions of $\mathrm{SL}_{n}(\mathbb{Z})$. We also explore applications to logic and set theory by considering Borel reducibility. In particular, we point out some of the extra challenges one faces when working in the purely set-theoretic (i.e., Borel) context, as opposed to the more familiar measure context.

Finally, in the last section, we use Ioana's theorem to give a self-contained and slightly streamlined proof of Thomas's theorem that the complexity of the isomorphism problem for torsion-free abelian groups of finite rank increases strictly with the rank.

\section{Rigidity via Cocycles}

We begin by introducing a slightly more expansive notion of orbit equivalence rigidity. If $\Gamma \curvearrowright X$ and $\Lambda \curvearrowright Y$ are arbitrary Borel actions of countable groups, then a function $f: X \rightarrow Y$ is said to be a homomorphism of orbits if $\Gamma x=\Gamma x^{\prime}$ implies $\Lambda f(x)=\Lambda f\left(x^{\prime}\right)$. It is said to be a homomorphism of actions if additionally there exists a homomorphism $\phi$ : $\Gamma \rightarrow \Lambda$ such that $f(\gamma x)=\phi(\gamma) f(x)$. (Note that these terms are not exactly standard.) Informally, we will say that $\Gamma \curvearrowright X$ is superrigid if whenever $\Lambda \curvearrowright Y$ is a free action and $f: X \rightarrow Y$ is a homomorphism of orbits, then $f$ in fact arises from a homomorphism of actions (i.e., $f$ is equivalent to a homomorphism of actions in a sense defined below).

Following Margulis and Zimmer, we will require the language of Borel cocycles to describe and prove superrigidity results. A cocycle is an object which is associated with a given

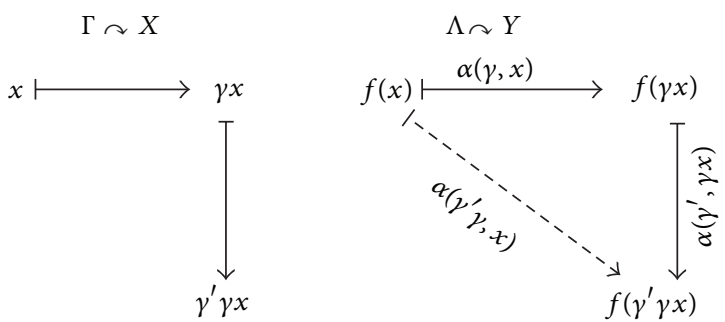

Figure 1: The cocycle condition: $\alpha\left(\gamma^{\prime} \gamma x\right)=\alpha\left(\gamma^{\prime}, \gamma x\right) \alpha(\gamma, x)$.

homomorphism of orbits $f: X \rightarrow Y$ as follows. Observe that for every $(\gamma, x) \in \Gamma \times X$, there exists a $\lambda \in \Lambda$ such that $f(\gamma x)=\lambda f(x)$. Moreover, $\Lambda$ acts freely on $Y$ if and only if this $\lambda$ is always uniquely determined by the data $f, \gamma$, and $x$. In other words, in this case, $f$ determines a function $\alpha: \Gamma \times X \rightarrow \Lambda$ which satisfies

$$
f(\gamma x)=\alpha(\gamma, x) f(x)
$$

This map is called the cocycle corresponding to $f$, and it is easy to see that it is Borel whenever $f$ is. Moreover, the cocycle $\alpha$ satisfies the composition law $\alpha\left(\gamma^{\prime} \gamma, x\right)=\alpha\left(\gamma^{\prime}, \gamma x\right) \alpha(\gamma, x)$; this is called the cocycle condition. See Figure 1 for a visual depiction of the cocycle condition.

When $f$ is actually action-preserving, that is, $f(\gamma x)=$ $\phi(\gamma) f(x)$ for some homomorphism $\phi: \Gamma \rightarrow \Lambda$, then we have $\alpha(\gamma, x)=\phi(\gamma)$, so that $\alpha$ is independent of the second coordinate. Conversely, if $\alpha$ is independent of the second coordinate, then one can define $\phi(\gamma)=\alpha(\gamma, \cdot)$ and the composition law implies that $\phi$ is a homomorphism. In this situation, the cocycle is said to be trivial.

In practice, when establishing rigidity, one typically shows that an arbitrary cocycle (arising from a homomorphism of orbits) is equivalent to a trivial cocycle (which therefore arises from a homomorphism of actions). Here, we say that homomorphisms of orbits $f, f^{\prime}: X \rightarrow Y$ are called equivalent if there exists a Borel function $b: X \rightarrow \Lambda$ such that $f^{\prime}(x)=b(x) f(x)$, a.e. (i.e., they lift the same function on the quotient spaces $X / \Gamma \rightarrow Y / \Lambda)$. In this case, the corresponding cocycles $\alpha, \alpha^{\prime}$ are said to be cohomologous. It is easy to check that $f, f^{\prime}$ are equivalent via $b$ if and only if the corresponding cocycles $\alpha, \alpha^{\prime}$ satisfy the relation $\alpha^{\prime}(\gamma, x)=$ $b(\gamma x) \alpha(\gamma, x) b(x)^{-1}$ a.e.; this is called the cohomology relation. The easiest way to see that this is the case is to glance at Figure 2.

We close this section by remarking that not all cocycles arise from orbit-preserving maps. An abstract cocycle is any Borel function satisfying the cocycle condition a.e., and two cocycles are said to be cohomologous if there exists a Borel function $b$ satisfying the cohomology relation a.e. The most powerful superrigidity results often have the conclusion that "every cocycle is cohomologous to a trivial cocycle." However, for most applications there is no need for the extra strength gained by using the abstract cocycle formulation. 


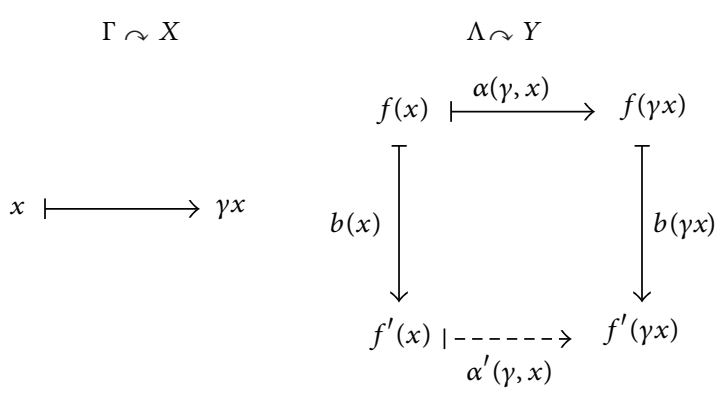

Figure 2: The cohomology relation for cocycles: $\alpha^{\prime}(\gamma, x)=$ $b(\gamma x) \alpha(\gamma, x) b(x)^{-1}$.

\section{Ioana's Theorem}

Cocycle superrigidity results were first established by Margulis and Zimmer for cocycles $\Gamma \curvearrowright X \rightarrow \Lambda$ where $\Gamma$ is a lattice in a higher rank Lie group acting ergodically on $X$. These results carried the additional hypothesis that $\Lambda$ is contained in an algebraic group. The first example of the most general form of cocycle superrigidity, with the target $\Lambda$ arbitrary, was Popa's result concerning Bernoulli actions. In this section, we will discuss Ioana's theorem, which establishes similar conclusions for profinite actions.

Here, $\Gamma \curvearrowright X$ is said to be profinite if as a $\Gamma$-set, $\mathrm{X}$ is the inverse limit of a family of finite $\Gamma$-sets $X_{n}$. In particular, there exist equivariant projections $\pi_{n}: X \rightarrow X_{n}$ and each element $x \in X$ can be identified with the thread $\left(\pi_{n}(x)\right)$. We are interested in the ergodic case; here, each $X_{n}$ is equipped with the uniform probability measure and $\Gamma \curvearrowright X_{n}$ is transitive.

Theorem 1 (Ioana). Let $\Gamma \curvearrowright(X, \mu)$ be an ergodic, measurepreserving, profinite action, with invariant factor maps $\pi_{n}$ : $X \rightarrow X_{n}$. Assume that $\Gamma$ has Property $(T)$. Then for any cocycle $\alpha: \Gamma \curvearrowright X \rightarrow \Lambda$, there exists $n$ and $a \in X_{n}$ such that the restriction of $\alpha$ to the action $\Gamma_{a} \curvearrowright \pi_{n}^{-1}(a)$ is cohomologous to a trivial cocycle.

In other words, the conclusion is that $\Gamma \curvearrowright X$ is "virtually superrigid" in the sense that any orbit preserving map, after it is restricted to a finite index component of the left-hand side, comes from an action preserving map. Ioana's theorem is interesting when contrasted with Popa's theorem; while Bernoulli actions are strongly mixing, profinite actions are highly nonmixing. Indeed, for each $n, \Gamma$ just permutes the blocks $\pi_{n}^{-1}(a)$, for $a \in X_{n}$, and it follows that $\bigcup_{a \in X_{n}} X_{a} \times X_{a}$ is a $\Gamma$-invariant subset of $X \times X$.

We remark that although our variant of Ioana's theorem is sufficient for most applications, it is weaker than the state of the art in several ways. First, Ioana requires only that $\Gamma$ have the relative Property $(\mathrm{T})$ over some infinite normal subgroups $N$ such that $\Gamma / N$ is finitely generated. Second, Ioana also shows that $\alpha$ is equivalent to a cocycle defined on all of $X$. Last, Furman has generalized the statement by replacing profinite actions with the more general class of compact actions. ( $\Gamma \curvearrowright X$ is said to be compact if when regarded as a subset of $(X, \mu)$ it is precompact in a suitable topology.)

\section{Cocycle Untwisting}

We begin with the following preliminary result, which roughly speaking says that if $\alpha: \Gamma \curvearrowright X \rightarrow \Lambda$ is a cocycle and if for each $\gamma$ it has a "very likely" value, then $\alpha$ is cohomologous to the map which always takes on this likely value. In particular, in this case, $\alpha$ is cohomologous to a trivial cocycle.

Theorem 2. Let $\Gamma \curvearrowright(X, \mu)$ be ergodic and measure-preserving, and let $\alpha: \Gamma \curvearrowright X \rightarrow \Lambda$ be a cocycle. Suppose that for all $\gamma \in \Gamma$ there exists $\lambda_{\gamma} \in \Lambda$ such that

$$
\mu\left\{x \mid \alpha(\gamma, x)=\lambda_{\gamma}\right\} \geq C>\frac{7}{8} .
$$

Then the map $\phi(\gamma)=\lambda_{\gamma}$ is a homomorphism and $\alpha$ is cohomologous to it.

It is easy to see that $\phi$ must be a homomorphism: indeed, the hypothesis guarantees that there is a nonnull set of $x$ for which $\phi\left(\gamma^{\prime} \gamma\right)=\alpha\left(\gamma^{\prime} \gamma, x\right)=\alpha\left(\gamma^{\prime}, \gamma x\right) \alpha(\gamma, x)=\phi\left(\gamma^{\prime}\right) \phi(\gamma)$. Hence it remains only to establish the following result.

Lemma 3. Let $\Gamma \curvearrowright(X, \mu)$ be ergodic and measure-preserving, and let $\alpha, \beta: \Gamma \curvearrowright X \rightarrow \Lambda$ be cocycles. Suppose that for all $\gamma \in \Gamma$

$$
\mu\{x \mid \alpha(\gamma, x)=\beta(\gamma, x)\} \geq C>\frac{7}{8} .
$$

Then $\alpha$ is cohomologous to $\beta$.

We understand this result to say that if $\alpha$ and $\beta$ are close in an $L^{\infty}$ sense, then they are cohomologous. It follows upon similar results of Popa and Furman, which draws the same conclusion in the case that $\alpha$ and $\beta$ are close in an appropriate $L^{1}$ sense (for instance, see [6, Theorem 4.2]). Ioana's proof, given below, may be safely skipped until reading the next section.

Proof of Lemma 3. Let $\Gamma \curvearrowright X \times \Lambda$ be the action given by

$$
\gamma(x, \lambda)=\left(\gamma x, \alpha(\gamma, x) \lambda \beta(\gamma, x)^{-1}\right)
$$

(this is an action thanks to the cocycle condition), and consider the corresponding left-regular representation. The reason for using this representation is that $\alpha$ is close to $\beta$ if and only if a particular vector is close to being invariant. Namely, let

$$
\xi=\chi_{X \times e}
$$

(read: the characteristic function of $X \times e$ ) and notice that

$$
\langle\gamma \xi, \xi\rangle=\mu\{x \mid \alpha(\gamma, x)=\beta(\gamma, x)\} .
$$

Using this together with the law of cosines, the hypothesis now translates to say that $\|\gamma \xi-\xi\| \leq C<1 / 2$ for all $\gamma \in \Gamma$. It is not difficult to see that this implies that there is an invariant vector $\eta$ such that $\|\eta-\xi\|<1 / 2$. (Indeed, letting $S$ denote 
the convex hull of $\overline{\Gamma \cdot \xi}$, it is easily seen that there exists a unique vector $\eta \in S$ of minimal norm; this $\eta$ is necessarily invariant.)

The idea for the conclusion of the proof is as follows. If we had $\eta=\chi_{\operatorname{graph}(b)}$ for some function $b: X \rightarrow \Lambda$, then we would be done. Indeed, in this case, the invariance of $\eta$ would mean that $b(x)=\lambda$ if and only if $b(\gamma x)=\alpha(\gamma, x) \lambda \beta(\gamma, x)^{-1}$, so that $b(\gamma x)=\alpha(\gamma, x) b(\gamma x) \beta(\gamma, x)^{-1}$. In other words, $b$ would witness the fact that $\alpha$ is cohomologous to $\beta$. The fact that $\|\eta-\xi\|<1 / 2$ implies that this is close to being the case.

We actually define $b(x)=$ the $\lambda$ such that $|\eta(x, \lambda)|>1 / 2$, if it exists and is unique. The above computation shows that when $b(x), b(\gamma x)$ are both defined, the cohomology relation holds. Moreover, the set where $b$ is defined is invariant, so by the ergodicity of $\Gamma \curvearrowright X$, it suffices to show that this set is nonnull. In fact, since $\eta$ and $\xi$ are close, $b$ must take value $e$ on a nonnull set:

$$
\begin{aligned}
\mu\{x & \left.:|\xi(x, e)-\eta(x, e)| \geq \frac{1}{2}\right\} \\
& \leq 4 \int_{\{x:|\xi(x, e)-\eta(x, e)| \geq 1 / 2\}}|\xi-\eta|^{2} \\
& \leq 4\|\xi-\eta\|^{2} \\
& <1 .
\end{aligned}
$$

This shows that $\{x:|\eta(x, e)|>1 / 2\}$ is non-null, as desired. A similar computation is used to show that, with probability 1 , $e$ is the unique such element of $\lambda$.

\section{Ioana's Proof}

What We Want. We wish to find some $n$ and $a \in X_{n}$ such that for all $\gamma \in \Gamma_{a}$,

$$
\left(\mu_{a} \times \mu_{a}\right)\left\{x, x^{\prime} \mid \alpha(\gamma, x)=\alpha\left(\gamma, x^{\prime}\right)\right\} \geq C>\frac{7}{8}
$$

where $\mu_{a}$ denotes the normalized restriction of $\mu$ to $\pi_{n}^{-1}(a)$. This would imply, by a straightforward computation, that for each $\gamma \in \Gamma_{a}$ there exists a $\lambda \in \Lambda$ such that

$$
\mu_{a}\{x \mid \alpha(\gamma, x)=\lambda\} \geq C>\frac{7}{8}
$$

and this would complete the proof thanks to Theorem 2.

What We Have. Unfortunately, it is only immediately possible to obtain that the quantities in (8) tend to 1 on average, at a rate depending on $\gamma$. That is, for each $\gamma \in \Gamma$, we have

$$
\lim _{n \rightarrow \infty} \frac{1}{\left|X_{n}\right|} \sum_{a \in X_{n}}\left(\mu_{a} \times \mu_{a}\right)\left\{x, x^{\prime} \mid \alpha(\gamma, x)=\alpha\left(\gamma, x^{\prime}\right)\right\}=1 .
$$

To see this, first note that it is equivalent to

$$
\lim _{n \rightarrow \infty} \sum_{\lambda \in \Lambda}\left(\frac{1}{\left|X_{n}\right|} \sum_{a \in X_{n}} \mu_{a}\{x \mid \alpha(\gamma, x)=\lambda\}^{2}\right)=1 .
$$

Now, we generally have that for any subset $S \subset X$,

$$
\lim _{n \rightarrow \infty} \frac{1}{\left|X_{n}\right|} \sum_{a \in X_{n}} \mu_{a}(S)^{2}=\mu(S) .
$$

This is because the family $\left\{\chi_{\pi_{n}^{-1}(a)} \mid a \in X_{n}, n \in \omega\right\}$ is dense in $L^{2}$, and while the right-hand side is the norm-squared of $\chi_{S}$, the left-hand side is the norm-squared of $\chi_{s}$ projected onto the span of $\left\{\chi_{\pi_{m}^{-1}(a)} \mid a \in X_{m}, m \leq n\right\}$. Finally, just apply (12) to each set $S=\{x \mid \alpha(\gamma, x)=\lambda\}$ and use the dominated convergence theorem to pass the limit through the sum over all $\lambda \in \Lambda$.

Proof. The gap between what he has (the asymptotic information) and what we want (the uniform information) is bridged by Property (T). Once again the first step is to consider an appropriate representation; this time one which compares the values of $\alpha(\gamma, x)$ as $x$ varies. That is, let $\Gamma \curvearrowright X \times X \times \Lambda$ by

$$
\gamma\left(x, x^{\prime}, \lambda\right)=\left(\gamma x, \gamma x^{\prime}, \alpha(\gamma, x) \lambda \alpha\left(\gamma, x^{\prime}\right)^{-1}\right)
$$

and consider the left-regular unitary representation corresponding to this action. The idea, very roughly, is that the degree to which $\alpha(\gamma, x)$ is independent of $x$ will be measured by how close a particular vector is to being $\Gamma$-invariant.

More precisely, for each $n$ define an orthonormal family of vectors $\xi_{a}$ for $a \in X_{n}$ by

$$
\xi_{a}=\left|X_{n}\right| \cdot \chi_{\pi_{n}^{-1}(a) \times \pi_{n}^{-1}(a) \times e},
$$

and consider their normalized average

$$
\xi_{n}=\frac{1}{\sqrt{\left|X_{n}\right|}} \sum_{a \in X_{n}} \xi_{a}
$$

Then a simple calculation shows that

$$
\begin{array}{r}
\left\langle\gamma \xi_{a}, \xi_{a}\right\rangle=\left(\mu_{a} \times \mu_{a}\right)\left\{x, x^{\prime} \mid \alpha(\gamma, x)=\alpha\left(\gamma, x^{\prime}\right)\right\}, \\
\left\langle\gamma \xi_{n}, \xi_{n}\right\rangle=\frac{1}{\left|X_{n}\right|} \sum_{a \in X_{n}}\left(\mu_{a} \times \mu_{a}\right)\left\{x, x^{\prime} \mid \alpha(\gamma, x)\right. \\
\left.=\alpha\left(\gamma, x^{\prime}\right)\right\} .
\end{array}
$$

So now, "what we have" and "what we want" can be translated as follows: we have the $\xi_{n}$ form a family of almost invariant vectors and we want a single $n$ and $a \in X_{n}$ such that $\xi_{a}$ is nearly invariant, uniformly for all $\gamma \in \Gamma_{a}$.

The remainder of the argument is straightforward. Since the $\xi_{n}$ forms a family of almost invariant vectors, Property (T) implies that there exist $n$ and an invariant vector $\eta$ such that $\left\|\eta-\xi_{n}\right\| \leq \delta$. Let $\eta^{\prime}$ be the restriction of $\eta$ to the set $\cup_{a \in X_{n}}\left(\pi_{n}^{-1}(a) \times \pi_{n}^{-1}(a) \times \Lambda\right)$. Since this set is invariant, we have the fact that that $\eta^{\prime}$ is invariant as well. Since $\xi_{n}$ is supported on this set, we retain the property that $\left\|\eta^{\prime}-\xi_{n}\right\| \leq \delta$.

Now, we simply express $\eta^{\prime}$ as a normalized average of orthogonal $\Gamma_{a}$-invariant vectors. More specifically, write

$$
\eta^{\prime}=\frac{1}{\sqrt{\left|X_{n}\right|}} \sum_{a \in X_{a}} \eta_{a}
$$


where $\eta_{a}$ is the appropriately rescaled restriction of $\eta^{\prime}$ to the set $\pi_{n}^{-1}(a) \times \pi_{n}^{-1}(a) \times \Lambda$. Then by the law of averages, we must have some $a \in X_{n}$ such that $\left\|\eta_{a}-\xi_{a}\right\| \leq \delta$. Moreover, $\eta_{a}$ is $\Gamma_{a^{-}}$ invariant, so that for all $\gamma \in \Gamma_{a}$ we have $\left\langle\gamma \eta_{a}, \eta_{a}\right\rangle=1$. It follows that by an appropriate choice of $\delta$, we can make $\left\langle\gamma \xi_{a}, \xi_{a}\right\rangle \geq$ $C>7 / 8$ for all $\gamma \in \Gamma_{a}$.

\section{Easy Applications}

In this section, we use Ioana's theorem for one of its intended purposes: to find many highly inequivalent actions. The results mentioned here are just meant to give the flavor of applications of superrigidity; they by no means demonstrate the full power of the theorem. In the next section we will discuss the slightly more interesting and difficult application to torsion-free abelian groups. For further applications, see for instance [7-10].

In searching for inequivalent actions, one might of course consider a variety of inequivalence notions. Here, we focus on just two of them: orbit inequivalence and Borel incomparability. Recall from the introduction that $\Gamma \curvearrowright X$ and $\Lambda \curvearrowright Y$ are said to be orbit equivalent if there exists a measurepreserving and orbit-preserving almost bijection from $X$ to $Y$. Notice that this notion depends only on the orbit equivalence relation arising from the two actions and not on the actions themselves. When this is the case, we will often conflate the two, saying alternately that certain actions are orbit equivalent or that certain equivalence relations are "orbit equivalent."

Borel bireducibility is a purely set-theoretic notion with its origins in logic. The connection is that if $E$ is an equivalence relation on a standard Borel space $X$, then we can think of $E$ representing a classification problem. For instance, if $X$ happens to be a set of codes for a family of structures, then studying the classification of those structures amounts to studying the isomorphism equivalence relation $E$ on $X$. We refer the reader to [11] for a complete introduction to the subject.

If $E$ and $F$ are equivalent relations on $X$ and $Y$, then $E$ is said to be Borel reducible to $F$ if there exists a Borel function $f: X \rightarrow Y$ satisfying $x E x^{\prime}$ if and only if $f(x) F f\left(x^{\prime}\right)$. We think of this saying that the classification problem for elements of $X$ up to $E$ is no more complex than the classification problem for elements of $Y$ up to $F$. Thus, if $E$ and $F$ are Borel bireducible (i.e., there is a reduction both ways), then they represent classification problems of the same complexity.

It is elementary to see that neither orbit equivalence or Borel bireducibility implies the other. For instance, given any $\Gamma$-space $X$ one can form a disjoint union $X \sqcup X^{\prime}$, where $X^{\prime}$ is a $\Gamma$-space of very high complexity which is declared to be of measure 0 . Conversely, if $X$ is an ergodic and hyperfinite $\Gamma$-space, then it is known that it is bireducible with $X \sqcup X$, but the two cannot be orbit equivalent. It is even possible, without much more difficulty, to find two ergodic actions which are bireducible but not orbit equivalent.

We are now ready to begin with the following direct consequence of Ioana's theorem. It was first established by Simon Thomas in connection working on classification problem for torsion-free abelian groups of finite rank. His proof used Zimmer's superrigidity theorem and some additional cocycle manipulation techniques; with Ioana's theorem in hand, the proof will be much simpler.

Corollary 4. If $n \geq 3$ is fixed and $p, q$ are primes such that $p \neq q$, then the actions of $S L_{n}(\mathbb{Z})$ on $S L_{n}\left(\mathbb{Z}_{p}\right)$ and $S L_{n}\left(\mathbb{Z}_{q}\right)$ are orbit inequivalent and Borel incomparable.

Here, $\mathbb{Z}_{p}$ denotes the ring of $p$-adic integers. It is easy to see that $\mathrm{SL}_{n}(\mathbb{Z}) \curvearrowright \mathrm{SL}_{n}\left(\mathbb{Z}_{p}\right)$ is a profinite action, being the inverse limit of the actions $\mathrm{SL}_{n}(\mathbb{Z}) \curvearrowright \mathrm{SL}_{n}\left(\mathbb{Z} / p^{i} \mathbb{Z}\right)$ together with their natural system of projections.

Proof. Let $p \neq q$ and suppose that $f$ is either an orbit equivalence or a Borel reduction from $\mathrm{SL}_{n}(\mathbb{Z}) \curvearrowright \mathrm{SL}_{n}\left(\mathbb{Z}_{p}\right)$ to $\mathrm{SL}_{n}(\mathbb{Z}) \curvearrowright \mathrm{SL}_{n}\left(\mathbb{Z}_{q}\right)$. We now apply Ioana's theorem together with the understanding of cocycles gained in the previous section. The conclusion is that we can suppose without loss of generality that there exists a finite index subgroup $\Gamma_{0} \leq$ $\mathrm{SL}_{n}(\mathbb{Z})$, a $\bar{\Gamma}_{0}$-coset $X \subset \mathrm{SL}_{n}\left(\mathbb{Z}_{p}\right)$, and a homomorphism $\phi: \Gamma_{0} \rightarrow \mathrm{SL}_{n}(\mathbb{Z})$ which makes $f$ into an action-preserving map from $\Gamma_{0} \curvearrowright X$ into $\mathrm{SL}_{n}(\mathbb{Z}) \curvearrowright \mathrm{SL}_{n}\left(\mathbb{Z}_{q}\right)$.

Now, in the measure-preserving case, it is not difficult to conclude that $f$ is a "virtual isomorphism" between the two actions. We claim that this can be achieved even in the case that $f$ is just a Borel reduction. First, we can assume that $\phi$ is an embedding. Indeed, by Margulis's theorem on normal subgroups [1, Theorem 8.1.2], either $\operatorname{im}(\phi)$ or $\operatorname{ker}(\phi)$ is finite. If $\operatorname{ker}(\phi)$ is finite, then we can replace $\Gamma_{0}$ by a finite index subgroup (and $X$ by a coset of the new $\bar{\Gamma}_{0}$ ) to suppose that $\phi$ is injective. On the other hand, if $\operatorname{im}(\phi)$ is finite, then we can replace $\Gamma_{0}$ by a finite index subgroup to suppose that $\phi$ is trivial. But this would mean that $f$ is $\Gamma_{0}$-invariant, and so by ergodicity of $\Gamma_{0} \curvearrowright X, f$ would send a conull set to a single point, contradicting that $f$ is countable-to-one.

Second, $\phi\left(\Gamma_{0}\right)$ must be a finite index subgroup of $\mathrm{SL}_{n}(\mathbb{Z})$. Indeed, by Margulis's superrigidity theorem, $\phi$ can be lifted to an isomorphism of $\mathrm{SL}_{n}(\mathbb{R})$, and it follows that $\phi\left(\Gamma_{0}\right)$ is a lattice of $\mathrm{SL}_{n}(\mathbb{R})$. But then it is easy to see that any lattice which is contained in $\mathrm{SL}_{n}(\mathbb{Z})$ must be commensurable with $\mathrm{SL}_{n}(\mathbb{Z})$.

Third, by the ergodicity of $\Gamma_{0} \curvearrowright X$, we can assume that $\operatorname{im}(f)$ is contained in a single $\overline{\phi\left(\Gamma_{0}\right)}$ coset $Y_{0}$. And now because $\phi\left(\Gamma_{0}\right)$ preserves a unique measure on $Y_{0}$ (the Haar measure) and because $\phi\left(\Gamma_{0}\right)$ preserves $f_{*}$ (Haar), we actually conclude that $f$ is measure-preserving. In summary, we have shown that $(\phi, f)$ is a measure and action-preserving isomorphism between $\Gamma_{0} \curvearrowright X_{0}$ and $\phi\left(\Gamma_{0}\right) \curvearrowright Y_{0}$, which establishes the claim.

Finally, a short computation confirms the intuitive, algebraic fact that the existence of such a map is ruled out by the mismatch in primes between the left-and right-hand sides. We give just a quick sketch; for a few more details see [12, Section 6]. Now, it is well-known that there are constants $A_{p}$ such that for any $\Delta \leq \mathrm{SL}_{n}(\mathbb{Z})$ of finite index, the index of $\bar{\Delta}$ in $\mathrm{SL}_{n}\left(\mathbb{Z}_{p}\right)$ divides $A_{p} p^{r}$ for some $r$. It follows that if $\Delta \leq \Gamma_{0}$, then $X$ breaks up into some number $N$ of ergodic $\Delta$-sets with $N \mid A_{p} p^{r}$. Since $(\phi, f)$ is a measure and action-preserving 
isomorphism, we also have that $Y$ breaks up into $N$ ergodic $\phi(\Delta)$ sets, and hence $N \mid A_{q} q^{s}$ also. But it is not difficult to choose $\Delta$ small enough to ensure that $N$ is large enough for this to be a contradiction.

This argument can be easily generalized to give uncountably many incomparable actions of $\mathrm{SL}_{n}(\mathbb{Z})$. Given an infinite set $S$ of primes with increasing enumeration $S=\left\{p_{i}\right\}$, we can construct a profinite $\mathrm{SL}_{n}(\mathbb{Z})$-set

$$
K_{S}=\operatorname{limSL}_{n}\left(\mathbb{Z} / p_{1} \cdots p_{i} \mathbb{Z}\right) .
$$

It is not much more difficult to show (as Ioana does) that when $\left|S \Delta S^{\prime}\right|=\infty$, the actions $\operatorname{SL}_{n}(\mathbb{Z}) \curvearrowright K_{S}$ and $\mathrm{SL}_{n}(\mathbb{Z}) \curvearrowright K_{S^{\prime}}$ are orbit inequivalent. In fact, this shows that there are " $E_{0}$ many" orbit inequivalent profinite actions of $\mathrm{SL}_{n}(\mathbb{Z})$. Of course, it is known from different arguments (exposited in [13, Theorem 17.1]) that the relation of orbit equivalence on the ergodic actions of $\mathrm{SL}_{n}(\mathbb{Z})$ is very complex (for instance not Borel). But the methods used here give us more detailed information: we have an explicit family of inequivalent actions, the actions are special (they are classical and profinite), and what is more they are Borel incomparable.

So far, we have considered only free actions of $\mathrm{SL}_{n}(\mathbb{Z})$. But if one just wants to use Ioana's theorem to find orbit inequivalent actions, it is enough to consider actions which are just free almost everywhere. Here, a measure-preserving action $\Gamma \curvearrowright X$ is said to be free almost everywhere if the set $\{x \mid \gamma \neq 1 \rightarrow \gamma x \neq x\}$ is conull (i.e., the set where $\Gamma$ acts freely is conull).

Unfortunately, in the purely Borel context it is not sufficient to work with actions which are free almost everywhere, since in this case we are not allowed to just delete a null set on the right-hand side. The next result shows how to get around this difficulty. Once again, it was originally obtained by Simon Thomas using Zimmer's superrigidity theorem.

Corollary 5. If $n \geq 3$ is fixed and $p, q$ are primes with $p \neq q$, then the actions of $S L_{n}(\mathbb{Z})$ on $\mathbb{P}\left(\mathbb{Q}_{p}^{n}\right)$ and $\mathbb{P}\left(\mathbb{Q}_{q}^{n}\right)$ are orbit inequivalent and Borel incomparable.

Here, $\mathbb{P}\left(\mathbb{Q}_{p}^{n}\right)$ denotes projective space of lines through $\mathbb{Q}_{p}^{n}$. Since $\mathbb{P}\left(\mathbb{Q}_{p}^{n}\right)$ is a transitive $\mathrm{SL}_{n}\left(\mathbb{Z}_{p}\right)$-space, this result is quite similar to the last one. We note also that while $\mathrm{SL}_{n}(\mathbb{Z})$ does not act freely on $\mathbb{P}\left(\mathbb{Q}_{p}^{n}\right)$, it does act freely on a conull subset [12, Lemma 6.2].

Proof. First suppose that $f: \mathbb{P}\left(\mathbb{Q}_{p}^{n}\right) \rightarrow \mathbb{P}\left(\mathbb{Q}_{q}^{n}\right)$ is a measurepreserving and orbit-preserving map. Then we can simply restrict the domain of $f$ to assume that it takes values in the part of $\mathbb{P}\left(\mathbb{Q}_{q}^{n}\right)$ where $\mathrm{SL}_{n}(\mathbb{Z})$ acts freely. Afterwards, we can obtain a contradiction using essentially the same combinatorial argument as in the proof of Corollary 4.

The proof in the case of Borel reducibility requires an extra step. Namely, we cannot be sure that $f$ sends a conull set into the part of $\mathbb{P}\left(\mathbb{Q}_{q}^{n}\right)$ where $\mathrm{SL}_{n}(\mathbb{Z})$ acts freely. However, if it does not, then by the ergodicity of $\operatorname{SL}_{n}(\mathbb{Z}) \curvearrowright \mathbb{P}\left(\mathbb{Q}_{p}^{n}\right)$ we can assume that $f$ sends a conull set into the part of $\mathbb{P}\left(\mathbb{Q}_{q}^{n}\right)$ where $\mathrm{SL}_{n}(\mathbb{Z})$ acts nonfreely. Our aim will be to show that this assumption leads to a contradiction.

First, let us assume that there exists a conull subset $X \subset$ $\mathbb{P}\left(\mathbb{Q}_{p}^{n}\right)$ such that, for all $x \in X$, there exists $\gamma \neq 1$ such that $\gamma f(x)=f(x)$. Then for all $x \in X, f(x)$ lies inside a nontrivial eigenspace of some element of $\mathrm{SL}_{n}(\mathbb{Z})$. Hence, if we let $V_{x}$ denote the minimal subspace of $\mathbb{Q}_{q}^{n}$ which is defined over $\frac{x}{\mathbb{Q}}$ such that $f(x) \subset V_{x}$, then $V_{x}$ is necessarily nontrivial.

Note that since $\overline{\mathbb{Q}}$ is countable, there are only countably many possibilities for $V_{x}$. Hence, there exists a non-null subset $X^{\prime}$ of $X$ and a fixed subspace $V$ of $\mathbb{Q}_{q}^{n}$ such that for all $x \in X^{\prime}$, we have $V_{x}=V$. By the ergodicity of $\mathrm{SL}_{n}(\mathbb{Z}) \curvearrowright X$, the set $X^{\prime \prime}=\mathrm{SL}_{n}(\mathbb{Z}) \cdot X^{\prime}$ is conull, and it follows that we can adjust $f$ to assume that for all $x \in X^{\prime \prime}$ we have $V_{x}=V$. (More precisely, replace $f(x)$ by $f^{\prime}(x)=f(\gamma x)$, where $\gamma$ is the first element of $\mathrm{SL}_{n}(\mathbb{Z})$ such that $\gamma x \in X^{\prime \prime}$.)

Now, let $H \leq \mathrm{GL}(V)$ denote the group of projective linear transformations induced on $V$ by $\mathrm{SL}_{n}(\mathbb{Z})_{\{V\}}$. It is an easy exercise, using the minimality of $V$, to check that $H$ acts freely on $\mathbb{P}(V)$ and that $f$ is a homomorphism of orbits from $\mathrm{SL}_{n}(\mathbb{Z}) \curvearrowright X^{\prime \prime}$ into $H \curvearrowright \mathbb{P}(V)$. Admitting this, we can finally apply Ioana's theorem to suppose that there exists a finite index subgroup $\Gamma_{0} \leq \Gamma$ and a nontrivial homomorphism $\phi: \Gamma_{0} \rightarrow H$. As in the proof of Corollary 4, we can suppose that $\phi$ is an embedding. We thus get a contradiction from the next result, below.

Theorem 6. If $\Gamma_{0} \leq S L_{n}(\mathbb{Z})$ is a subgroup of finite index and $\mathbf{G}$ is an algebraic $\overline{\mathbb{Q}}$-group with $\operatorname{dim}(\mathbf{G})<n^{2}-1$, then $\Gamma_{0}$ does not embed $\mathbf{G}(\overline{\mathbb{Q}})$.

The idea of the proof is to apply Margulis's superrigidity theorem. That is, one wishes to conclude that such an embedding lifts to some kind of rational map $\mathrm{SL}_{n}(\mathbb{R}) \rightarrow \mathbf{G}$, a clear dimension contradiction. However, a little extra work is needed to handle the case of a $\overline{\mathbb{Q}}$-group on the right-hand side (see [10, Theorem 4.4]).

\section{Torsion-Free Abelian Groups of Finite Rank}

The torsion-free abelian groups of rank 1 were classified by Baer in 1937. The next year, Kurosh and Malcev expanded on his methods to give classifications for the torsion-free abelian groups of ranks 2 and higher. Their solution, however, was considered inadequate because the invariants they provided were no easier to distinguish than the groups themselves.

In 1998, Hjorth proved, using methods from the study of Borel equivalence relations, that the classification problem for rank 2 torsion-free abelian groups is strictly harder than that for rank 1 (see [14]). However, his work did not answer the question of whether the classification problem for rank 2 groups is as complex as for all finite ranks or whether there is more complexity that is to be found by looking at ranks 3 and higher.

Let $R(n)$ denote the space of torsion-free abelian groups of rank exactly $n$, that is, the set of full-rank subgroups of $\mathbb{Q}^{n}$. Let $\cong_{n}$ denote the isomorphism relation on $R(n)$. In this section 
we will give a concise and essentially self-contained proof of Thomas's theorem:

Theorem 7 (Thomas [15]). For $n \geq 2$, one has that $\cong_{n}$ lies properly below $\cong_{n+1}$ in the Borel reducibility order.

Thomas's original argument used Zimmer's superrigidity theorem. In this presentation, we have essentially copied his argument verbatim, with a few simplifications stemming from the use of Ioana's theorem instead of Zimmer's theorem.

The first connection between this result and the results of the last section is that for $A, B \in R(n)$, we have $A \cong B$ if and only if there exists $g \in \mathrm{GL}_{n}(\mathbb{Q})$ such that $B=g(A)$. Hence, the isomorphism relation $\cong_{n}$ is given by a natural action of the linear group $\mathrm{GL}_{n}(\mathbb{Q})$. Unfortunately, even restricting to just the action of $\mathrm{SL}_{n}(\mathbb{Z})$, the space $R(n)$ is nothing like a profinite space.

The Kurosh-Malcev Invariants. Although I have said that the Kurosh-Malcev invariants do not adequately classify the torsion-free abelian groups of finite rank, we will get around our difficulties by working with the Kurosh-Malcev invariants rather than with the original space $R(n)$. The following is the key result concerning the invariants; see [16, Chapter 93] for a full account.

Theorem 8 (Kurosh, Malcev). The map $A \mapsto A_{p}=\mathbb{Z}_{p} \otimes A$ is a $G L_{n}(\mathbb{Q})$-preserving bijection between the (full rank) p-local subgroups of $\mathbb{Q}^{n}$ and the (full rank) $\mathbb{Z}_{p}$-submodules of $\mathbb{Q}_{p}^{n}$. The inverse map is given by $A_{p} \mapsto A=A_{p} \cap \mathbb{Q}^{n}$.

Here, a subgroup of $\mathbb{Q}^{n}$ is said to be $p$-local if it is infinitely $q$-divisible for each prime $q \neq p$. Kurosh and Malcev proved that a subgroup $A \leq \mathbb{Q}^{n}$ is determined by the sequence $\left(A_{p}\right)$; this sequence is said to be the Kurosh-Malcev invariant corresponding to $A$. It follows of course that $A$ is determined up to isomorphism by the orbit of $\left(A_{p}\right)$ under the coordinatewise action of $\mathrm{GL}_{n}(\mathbb{Q})$. (It is now easy to see why these invariants serve as a poor classification: such orbits can be quite complex.) All that we will need from this classification is the following corollary.

Proposition 9. There exists a Borel reduction from $G L_{n}(\mathbb{Q}) \curvearrowright$ $\mathbb{P}\left(\mathbb{Q}_{p}^{n}\right)$ to $\cong_{n}$.

Since $\mathrm{GL}_{n}(\mathbb{Q}) \curvearrowright \mathbb{P}\left(\mathbb{Q}_{p}^{n}\right)$ is closely related to a profinite action, Proposition 9 will eventually enable us to apply Ioana's theorem in the proof of Theorem 7.

Sketch of Proof. Given a linear subspace $V \leq \mathbb{Q}_{p}^{n}$, let $V^{\perp}$ denote its orthogonal complement. Then there exists a vector $v$ such that $V^{\perp} \oplus \mathbb{Z}_{p} v$ is a full-rank submodule of $\mathbb{Q}_{p}^{n}$. By Theorem 8, this module corresponds to an element $f(V) \in$ $R(n)$. This is how the Kurosh-Malcev construction is used.

To verify that it works, one uses the fact that the KuroshMalcev construction is $\mathrm{GL}_{n}(\mathbb{Q})$-preserving, together with the technical fact: if $\operatorname{dim} W=\operatorname{dim} W^{\prime}=n-1$ and $W \oplus \mathbb{Z}_{p} w, W^{\prime} \oplus$ $\mathbb{Z}_{p} w^{\prime}$ are full-rank modules, then $W^{\prime}=g W$ for some $g \in \mathrm{GL}_{n}(\mathbb{Q})$ actually implies that $W^{\prime} \oplus \mathbb{Z}_{p} w^{\prime}=g\left(W \oplus \mathbb{Z}_{p} w\right)$ for some $g \in \mathrm{GL}_{n}(\mathbb{Q})$.

The Problem of Freeness. Suppose now that $n \geq 2$ and that there exists a Borel reduction from $\cong_{n+1}$ to $\simeq_{n}$. By Proposition 9, there exists a profinite, ergodic $\mathrm{SL}_{n+1}(\mathbb{Z})$-space $X$ (namely $X=\mathbb{P}\left(\mathbb{Q}_{p}^{n+1}\right)$ ) and a countable-to-one homomorphism of orbits $f$ from $\mathrm{SL}_{n+1}(\mathbb{Z}) \curvearrowright X$ to $\cong_{n}$. We can almost apply Ioana's theorem, except that unfortunately $\cong_{n}$ is not induced by a free action of any group. The following simple observation gives us an approach for getting around this difficulty.

Proposition 10. Let $f$ be a homomorphism of orbits from $\Gamma \curvearrowright$ $X$ into $\Lambda \curvearrowright Y$. Suppose that there exists a fixed $K \leq \Lambda$ such that for all $x \in X, \operatorname{stab}_{\Lambda}(f(x))=K$. Then $N_{\Lambda}(K) / K$ acts freely on $f(X)$, and $f$ is a homomorphism of orbits from $\Gamma \curvearrowright X$ into $N_{\Lambda}(K) / K \curvearrowright f(X)$.

Proof. By definition, we have that $N_{\Lambda}(K) / K$ acts on $f(X)$ by $\lambda K \cdot y=\lambda y$. The action is free because $\lambda y=y$ implies that $\lambda \in K$. To see that $f$ is still a homomorphism of orbits, just note that if $f\left(x^{\prime}\right)=\lambda f(x)$ then since $\operatorname{stab} f(x)=\operatorname{stab} f\left(x^{\prime}\right)=$ $K$, it follows that $\lambda$ normalizes $K$.

One can now formulate a strategy for proving Thomas's theorem along the following lines:

Claim 1. By passing to a conull subset of $X$, we can assume without loss of generality that for all $x$ we have $\operatorname{stab}_{\mathrm{GL}_{n}(\mathbb{Q})}(f(x))=$ some fixed $K$.

Claim 2. There cannot exist a nontrivial homomorphism from (a finite index subgroup of) $\mathrm{SL}_{n+1}(\mathbb{Z})$ into $N_{\mathrm{GL}_{n}(\mathbb{Q})}(K) /$ $K$.

This would yield a contradiction, since by Proposition 9 and Claim 1, Ioana's theorem would provide the nontrivial homomorphism ruled out in Claim 2. Unfortunately, this approach does not turn out to be a good one. The reason is that Claim 1 seems to be as difficult to be proved as Theorem 7 itself. Moreover, Claim 2 is not known to be true in this generality. (In fact, Claim 1 has recently been established by Thomas in [10], but his proof actually requires all of the arguments below and more).

Use Quasi-Isomorphism Instead. To reduce the number of possibilities for $\operatorname{stab}(f(x))=\operatorname{Aut}(f(x))$, we change categories from isomorphism to quasi-isomorphism. We say that groups $A, B \leq \mathbb{Q}^{n}$ are quasi-isomorphic, written as $A \sim_{n} B$, if and only if $B$ is commensurable with an isomorphic copy of $A$. Of course, $\sim_{n}$ is a courser relation than $\cong_{n}$, but it is easy to check that it is still a countable Borel equivalence relation (indeed, the commensurability relation is a countable relation in this case, see [15, Lemma 3.2]). Hence, the map $f$ from above is again a countable-to-one Borel homomorphism from $\mathrm{SL}_{n+1}(\mathbb{Z}) \curvearrowright X$ to $\sim_{n}$.

Now, rather than attempting to fix the automorphism group of $f(x)$, we will fix the quasiendomorphism ring 
QEnd $(A)$ of $f(x)$. Here, if $A \leq \mathbb{Q}^{n}$ then $g \in \mathrm{GL}_{n}(\mathbb{Q})$ is said to be a quasiendomorphism of $A$ if $\phi(A)$ is commensurable with a subgroup of $A$. (Equivalently, $n \phi(A) \subset A$ for some $n \in \mathbb{N}$.) Then unlike $\operatorname{End}(A)$, it is clear that $\operatorname{QEnd}(A)$ is a $\mathbb{Q}$-subalgebra of $M_{n \times n}(\mathbb{Q})$. It follows that there are just countably many possibilities for QEnd $(f(x))$, since an algebra is determined by any $\mathbb{Q}$-vector space basis for it. Hence, there exists $K$ such that QEnd $(f(x))=K$ for a nonnull set of $x$. Arguing as in the proof of Corollary 5, we may replace $X$ by a conull subset and adjust $f$ to assume that for all $x \in X$, we have QEnd $(f(x))=K$.

Thus, we have successfully obtained our analog of Claim 1 for quasi-isomorphism. Indeed, copying the arguments in the proof of Proposition 10, we see that $f$ is a homomorphism

$$
f: \mathrm{SL}_{n+1}(\mathbb{Z}) \curvearrowright X \rightarrow \frac{N_{\mathrm{GL}_{n}(\mathbb{Q})}(K)}{K^{\times} \curvearrowright f(X)}
$$

and that $N_{\mathrm{GL}_{n}(\mathbb{Q})}(K) / K^{\times}$acts freely on $f(X)$. We may therefore apply Ioana's theorem to suppose that there exists a finite index subgroup $\Gamma_{0} \leq \mathrm{PSL}_{n+1}(\mathbb{Z})$, a positive measure $X_{0} \subset X$, and a homomorphism $\phi: \Gamma_{0} \rightarrow N_{\mathrm{GL}_{n}(\mathbb{Q})}(K) / K^{\times}$ such that for $x \in X_{0}$ and $\gamma \in \Gamma$, we have

$$
f(\gamma x)=\phi(\gamma) f(x) .
$$

Note that $\phi$ must be nontrivial; since if $\phi\left(\Gamma_{0}\right)=1$, then this says that $f$ is $\Gamma_{0}$-invariant. But then, by ergodicity of $\Gamma_{0} \curvearrowright X_{0}$, $f$ would send a conull set to one point, contradicting that $f$ is countable-to-one.

A Dimension Contradiction. The set theory is now over; we have only to establish the algebraic fact that the analog of Claim 2 holds: there does not exist a nontrivial homomorphism from $\Gamma_{0}$ into $N_{\mathrm{GL}_{n}(\mathbb{Q})}(K) / K^{\times}$. Again by Margulis's theorem on normal subgroups, we can suppose that $\phi$ is an embedding. Then using Margulis's superrigidity theorem, it suffices to show that $N_{\mathrm{GL}_{n}(\mathbb{Q})}(K) / K^{\times}$is contained in an algebraic group of dimension strictly smaller than $\operatorname{dim}\left(\mathbf{P S L}_{n+1}\right)=$ $(n+1)^{2}-1$.

To see this, first note that since the subalgebra $K$ of $M_{n \times n}(\mathbb{Q})$ is definable from a vector space basis, we have that $K=\mathbf{K}(\mathbb{Q})$, where $\mathbf{K}$ is an algebraic $\mathbb{Q}$-group inside $M_{n \times n}$. Basic facts from algebraic group theory imply that $N_{\mathrm{GL}_{n}(\mathbb{Q})}(K)=\mathbf{N}(\mathbb{Q})$ and $K^{\times}=\mathbf{K}^{\prime}(\mathbb{Q})$, where again $\mathbf{N}, \mathbf{K}^{\prime}$ are algebraic $\mathbb{Q}$-groups inside $M_{n \times n}$. Finally, $N_{\mathrm{GL}_{n}(\mathbb{Q})}(K) / K^{\times}$ is exactly $\mathbf{N}(\mathbb{Q}) / \mathbf{K}^{\prime}(\mathbb{Q})$, which is contained in the algebraic $\mathbb{Q}$-group $\mathbf{N} / \mathbf{K}^{\prime}$. Since the dimension of an algebraic group decreases when passing to subgroups and quotients, we have

$$
\operatorname{dim}\left(\frac{\mathbf{N}}{\mathbf{K}^{\prime}}\right) \leq \operatorname{dim}\left(M_{n \times n}\right)=n^{2}<(n+1)^{2}-1,
$$

as desired. This completes the proof.

\section{References}

[1] R. J. Zimmer, Ergodic Theory and Semisimple Groups, vol. 81 of Monographs in Mathematics, Birkhäuser, Basel, Switzerland, 1984.
[2] A. Furman, "Orbit equivalence rigidity," Annals of Mathematics. Second Series, vol. 150, no. 3, pp. 1083-1108, 1999.

[3] N. Monod and Y. Shalom, "Orbit equivalence rigidity and bounded cohomology," Annals of Mathematics, vol. 164, no. 3, pp. 825-878, 2006.

[4] Y. Kida, "Orbit equivalence rigidity for ergodic actions of the mapping class group," Geometriae Dedicata, vol. 131, pp. 99-109, 2008.

[5] S. Popa, "Cocycle and orbit equivalence superrigidity for malleable actions of $\omega$-rigid groups," Inventiones Mathematicae, vol. 170, no. 2, pp. 243-295, 2007.

[6] A. Furman, “On Popa's cocycle superrigidity theorem," International Mathematics Research Notices. IMRN, no. 19, 2007.

[7] A. Ioana, "Cocycle superrigidity for profinite actions of property (T) groups," Duke Mathematical Journal, vol. 157, no. 2, pp. 337-367, 2011.

[8] S. Coskey, "The classification of torsion-free abelian groups of finite rank up to isomorphism and up to quasi-isomorphism," Transactions of the American Mathematical Society, vol. 364, no. 1, pp. 175-194, 2012.

[9] S. Coskey, "Borel reductions of profinite actions of $S L_{n}(\mathbb{Z})$," Annals of Pure and Applied Logic, vol. 161, no. 10, pp. 1270-1279, 2010.

[10] S. Thomas, "The classification problem for $\delta$-local torsion-free abelian groups of finite rank," Advances in Mathematics, vol. 226, no. 4, pp. 3699-3723, 2011.

[11] S. Gao, Invariant Descriptive Set Theory, vol. 293 of Pure and Applied Mathematics, CRC Press, Boca Raton, Fla, USA, 2009.

[12] S. Thomas, "Superrigidity and countable Borel equivalence relations," Annals of Pure and Applied Logic, vol. 120, no. 1-3, pp. 237-262, 2003.

[13] A. S. Kechris, Global Aspects of Ergodic Group Actions, vol. 160 of Mathematical Surveys and Monographs, American Mathematical Society, Providence, RI, USA, 2010.

[14] G. Hjorth, "Around nonclassifiability for countable torsion free abelian groups," in Abelian Groups and Modules, pp. 269-292, Birkhäuser, Basel, Switzerland, 1999.

[15] S. Thomas, "The classification problem for torsion-free abelian groups of finite rank," Journal of the American Mathematical Society, vol. 16, no. 1, pp. 233-258, 2003.

[16] L. Fuchs, Infinite Abelian Groups, Academic Press, New York, NY, USA, 1973, Pure and Applied Mathematics. Vol. 36-II. 


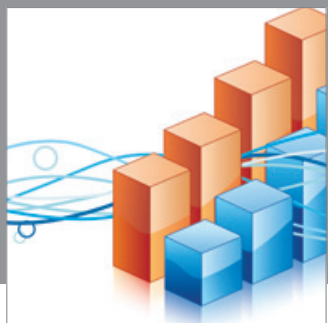

Advances in

Operations Research

mansans

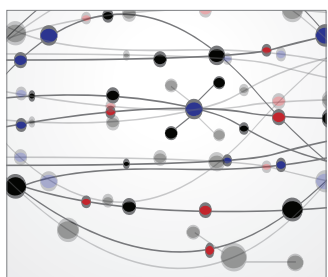

The Scientific World Journal
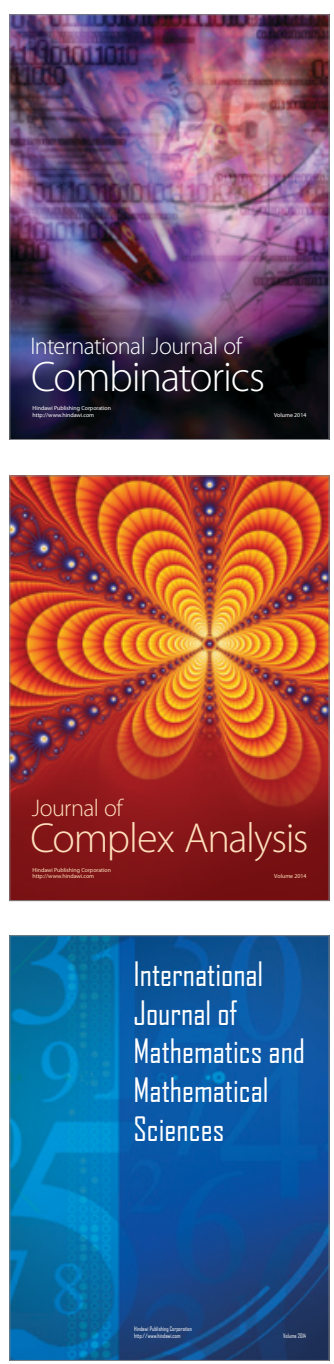
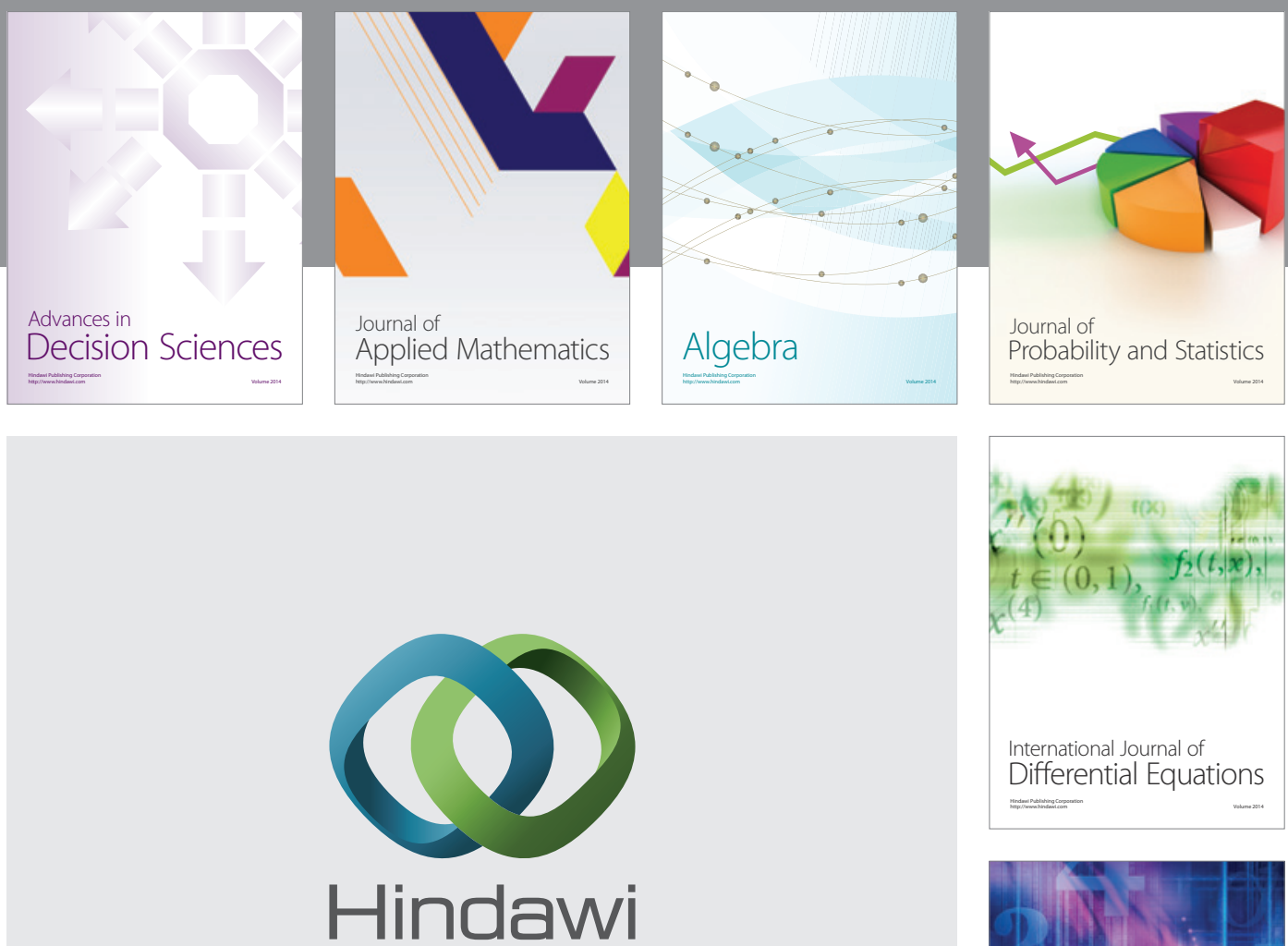

Submit your manuscripts at http://www.hindawi.com
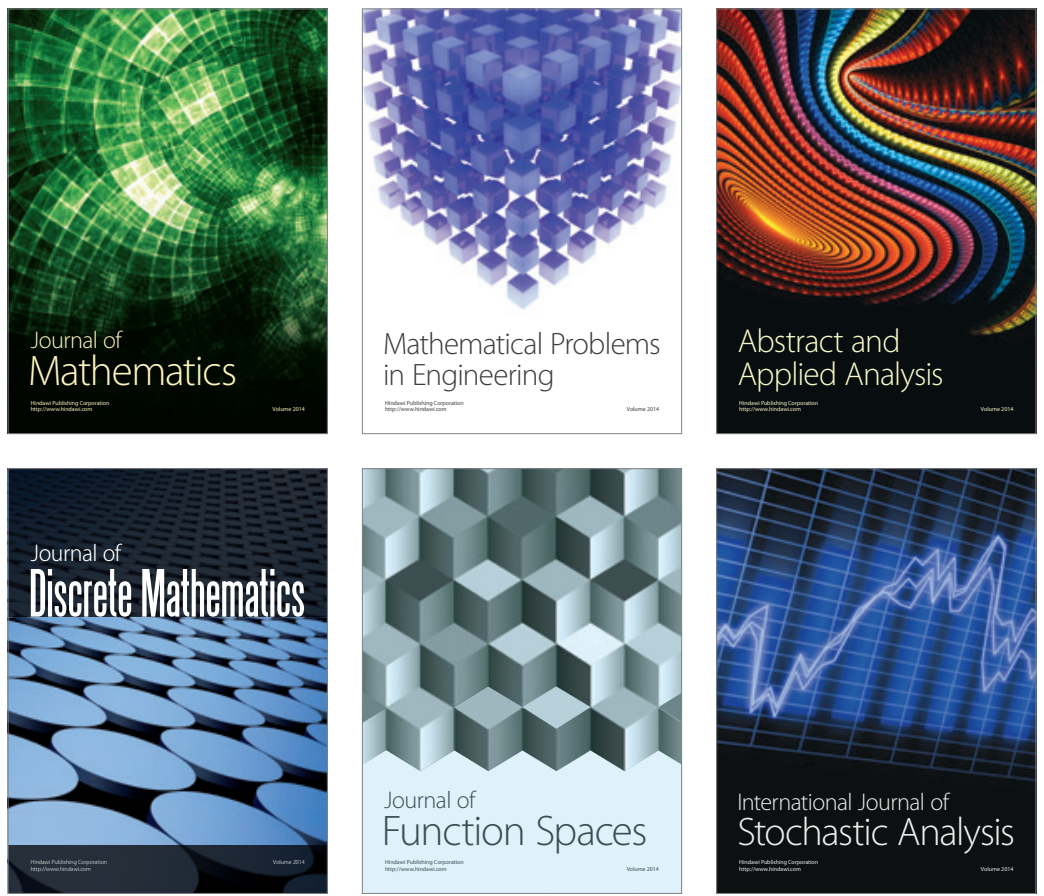

Journal of

Function Spaces

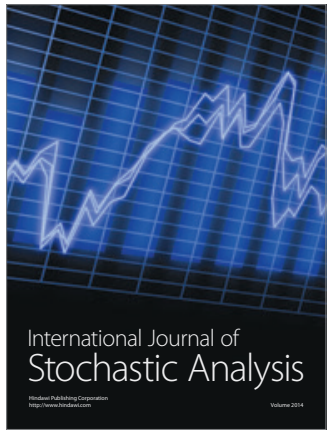

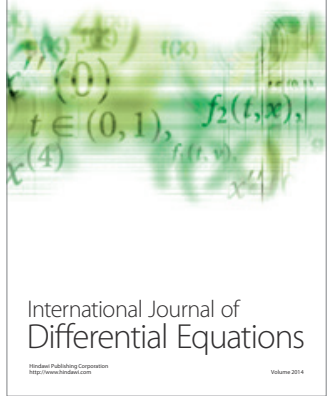
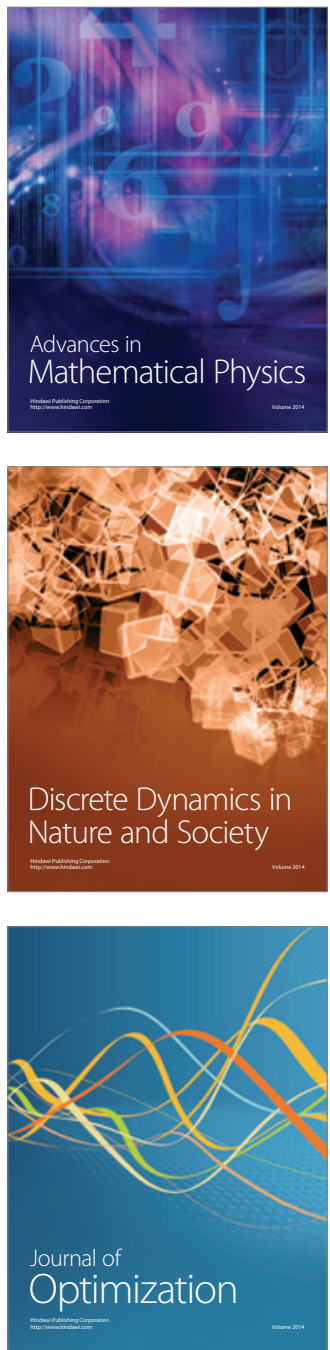